\title{
Aperçu
}

\section{Quel est l'état de santé des Canadiens? Brève mise à jour}

\author{
Comité directeur des IMCC*
}

\section{Présentation}

Le présent aperçu vise à mettre à jour un certain nombre de résultats clés tirés du rapport de 2016 "Quel est l'état de santé des Canadiens? $»^{1}$ en utilisant les données de la plus récente version (2018) des Indicateurs des maladies chroniques au Canada (IMCC; tableau 1). Le rapport de 2016 examinait les tendances liées aux quatre maladies chroniques majeures (maladies cardiovasculaires $[\mathrm{MCV}]^{\dagger}$, cancer, maladies respiratoires chroniques $[\mathrm{MRC}]^{\ddagger}$ et diabète) et quatre facteurs de risque sous-jacents (tabagisme, inactivité physique, mauvaise alimentation et consommation nocive d'alcool) qui sont les fondements du Plan d'action mondial pour la lutte contre les maladies non transmissibles 2013-2020 de l'Organisation mondiale de la Santé (OMS) [Global action plan for the prevention and control of noncommunicable diseases 2013$2020]^{2}$. Le rapport de 2016 traitait aussi des troubles anxieux ou de l'humeur en raison de l'impact considérable de ces maladies sur la santé des Canadiens. Par conséquent, la présente mise à jour n'est pas axée sur tous les résultats des IMCC de 2018 indiqués dans le tableau 1, mais se concentre plutôt sur des éléments d'intérêt particuliers, notamment les expériences de morbidité et la mortalité des Canadiens vivant avec ces maladies chroniques majeures ainsi que les facteurs de risque connexes. Les résultats rapportés sont fondés sur les quatre sources de données suivantes : le Système canadien de surveillance des maladies chroniques (SCSMC); l'Enquête sur la santé dans les collectivités canadiennes
(ESCC); l'Enquête canadienne sur les mesures de la santé; et les Statistiques de l'état civil - Base canadienne de données sur les décès.

\section{Principales constatations}

\section{Mortalité}

Alors que le taux de mortalité associé aux quatre maladies chroniques majeures a diminué du tiers sur une période de 18 ans (passant de 663/100 000 en 1998 à 441/100 000 en 2015; taux de mortalité normalisés selon l'âge), la diminution la plus importante a été observée pour les MCV (près de $50 \%$ ). Néanmoins, les MCV sont toujours la seconde cause de mortalité en importance au Canada (196/100 000; taux brut de mortalité) après le cancer (215/100 000; taux brut de mortalité), représentant $27 \%$ et $29 \%$ de tous les décès en 2015, respectivement. En outre, la probabilité de mourir entre 30 et 69 ans (c.-à-d. mortalité prématurée) de l'une des 4 maladies chroniques majeures a diminué de près du tiers pendant la même période (passant de 14,9 \% en 1998 à 10,2 \% en 2015). Bien qu'il s'agisse d'un résultat positif, il reste encore beaucoup de travail à faire puisque les décès dus à ces maladies chez les Canadiens de ce groupe d'âge sont souvent évitables.

\section{Morbidité}

Un adulte canadien sur trois $(33,7 \%)$ est atteint d'au moins une des maladies chroniques suivantes : MCV; cancer; MRC;

\section{Diffuser cet article sur Twitter}

diabète; troubles anxieux ou de l'humeur. Plus précisément, en 2015-2016, environ 2,4 millions de Canadiens vivaient avec une cardiopathie ischémique et environ 800000 Canadiens vivaient avec les conséquences d'un accident vasculaire cérébral, deux formes communes de MCV. Environ 2,2 millions de Canadiens ont déclaré avoir déjà reçu un diagnostic de cancer. De plus, 2,1 millions de Canadiens vivaient avec une maladie pulmonaire obstructive chronique (MPOC), une des MRC les plus fréquentes, et 3,1 millions de Canadiens souffraient de diabète. Enfin, 3,9 millions de Canadiens ont affirmé avoir déjà reçu un diagnostic de troubles anxieux ou de l'humeur en $2016^{\S}$. À l'opposé de la plupart des maladies chroniques dont la fréquence augmente avec l'âge, les troubles anxieux ou de l'humeur sont particulièrement présents au sein de la population en âge de travailler (20 à 64 ans).

Le nombre de personnes vivant avec ces maladies chroniques continue d'augmenter en raison du vieillissement ainsi que de la croissance de la population canadienne et $\mathrm{du}$ fait que les gens vivent plus longtemps avec ces maladies grâce aux progrès réalisés en matière de traitement et de gestion des patients (figure 1). D’un autre côté, le taux de nouveaux cas diagnostiqués chaque année pour la plupart des maladies diminue lentement, sauf en ce qui concerne le diabète, pour lequel on a observé une plus grande variation des tendances*. La majorité des maladies chroniques touchent les hommes et les femmes différemment, certaines étant assurément plus

\footnotetext{
* Brenda Branchard, Paromita Deb-Rinker, Alejandra Dubois, Pam Lapointe, Siobhan O’Donnell, Louise Pelletier (présidente), Gabriela Williams.

+ Les MCV comprennent la cardiopathie ischémique, l'insuffisance cardiaque et les accidents vasculaires cérébraux (AVC).

† Les MRC comprennent la maladie pulmonaire obstructive chronique (MPOC) et l'asthme.

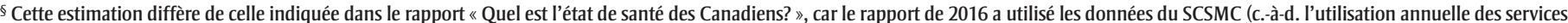
de santé pour les troubles anxieux et de l'humeur), alors que les IMCC ont utilisé les données de l'ESCC (c.à-d. les troubles de l'anxiété ou de l'humeur diagnostiqués autodéclarés).

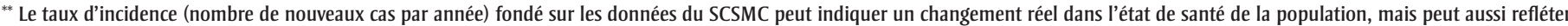
une évolution des méthodes de collecte des données, des systèmes de codage ou de classification, des pratiques cliniques, des méthodes de facturation, etc.
} 
FIGURE 1

Prévalence normalisée selon l'âge (\%) des maladies chroniques majeures chez les Canadiens âgés de 20 ans et plus, Canada, 2000 à 2015

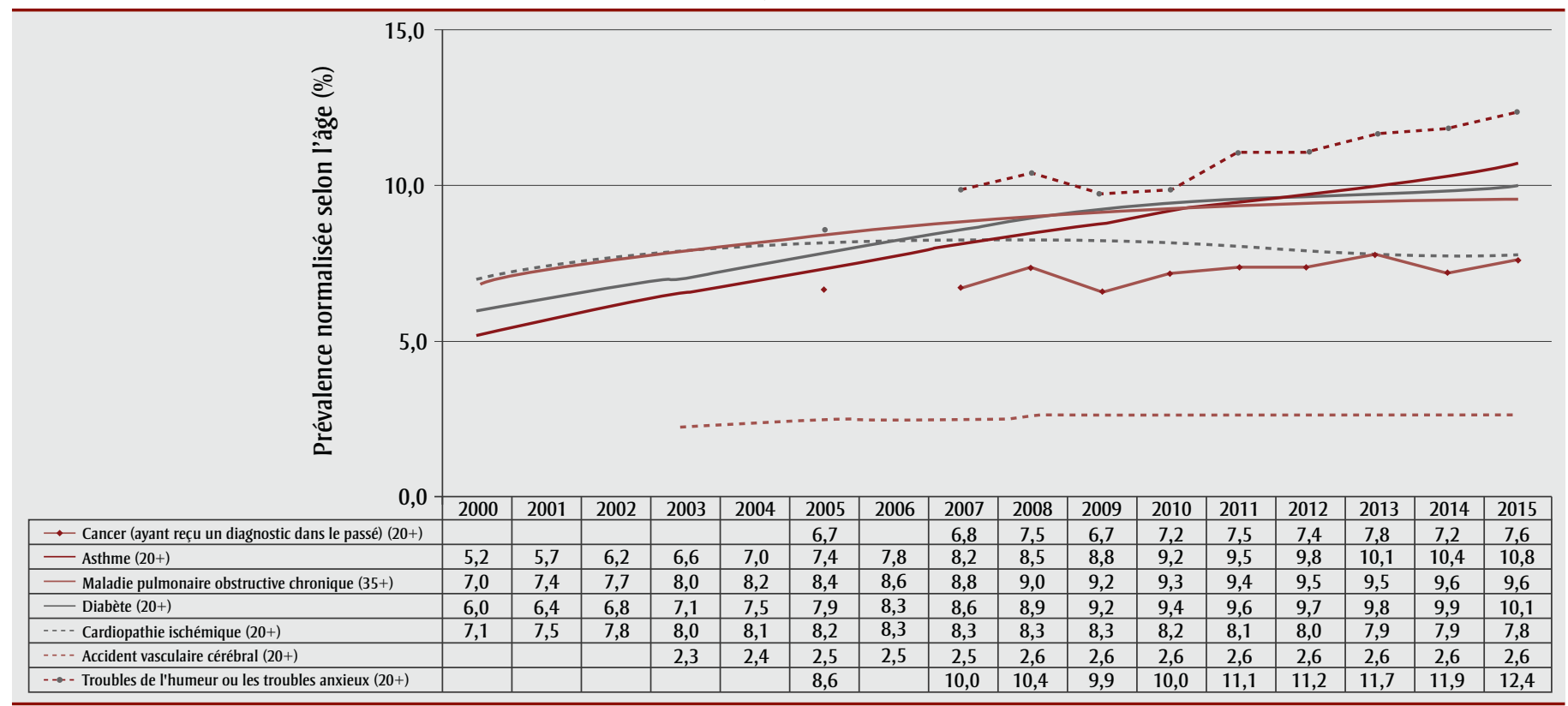

Sources : Enquête sur la santé dans les collectivités canadiennes - Composante annuelle, 2005 à 2015; Système canadien de surveillance des maladies chroniques, $2000-2001$ à 2015-2016.

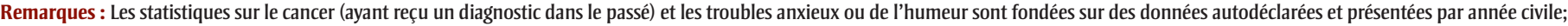
pour toutes les autres maladies et affections, les statistiques sont fondées sur des données administratives et sont présentées par exercice financier.

fréquentes chez les hommes (comme les cardiopathies ischémiques) et d'autres chez les femmes (comme les troubles anxieux ou de l'humeur). Dans l'ensemble, les femmes tendent à adopter de meilleurs comportements en matière de santé que les hommes, sauf en ce qui concerne l'activité physique, dont le niveau est bas pour tous les Canadiens. D'un autre côté, les femmes vivent plus longtemps et l'âge est un des principaux facteurs de risque non modifiables associés aux maladies chroniques.

\section{Facteurs de risque comportementaux}

Comme le mentionnait le rapport de 2016, sauf en ce qui a trait au tabagisme, le Canada continue d'observer une forte prévalence des facteurs de risque évitables associés aux maladies chroniques (inactivité physique $[62,4 \%$ pour les enfants et les jeunes et $82,5 \%$ pour les adultes], mauvaise alimentation [70 \% pour les Canadiens âgés de 12 ans et plus] et consommation nocive d'alcool [15,2 \% des Canadiens âgés de 15 ans et plus]). En 2016, 84,9 \% des adultes canadiens déclaraient présenter au moins un de ces facteurs de risque comportementaux.
Les maladies chroniques se développent généralement pendant de nombreuses années, voire des décennies. Il est donc très important que les enfants et les jeunes canadiens adoptent des comportements sains dès leur plus jeune âge. Malheureusement, seulement 1 enfant sur 10 (9,5\%) âgé entre 5 et 17 ans respecte les récentes Directives canadiennes en matière de mouvement sur 24 heures à l'intention des enfants et des jeunes relativement à l'activité physique, au comportement sédentaire et au sommeil ${ }^{3}$. De plus, seulement 1 enfant ou jeune sur $3(37,6 \%)$ respecte les Directives canadiennes en matière d'activité physique $^{\dagger+}$, ce qui indique que des efforts doivent encore être déployés pour augmenter les activités ainsi que le soutien aux sports et loisirs, au transport actif et à l'activité physique à l'école.

\section{Conclusion}

Depuis la publication du rapport de 2016, les tendances relatives à la mortalité globale et prématurée ont continué de diminuer à une vitesse variable pour les quatre principales maladies chroniques. Cependant, le nombre de Canadiens vivant avec au moins une de ces maladies chroniques continue d'augmenter, représentant un lourd fardeau pour la santé des Canadiens et le système de soins de santé. Les maladies chroniques traitées dans la présente mise à jour constituent les principales causes de décès et de morbidité au Canada et pourraient être évitées en grande partie en nous attaquant seulement à quelques facteurs de risque courants. Les modes de vie sains, la prévention et la bonne gestion des maladies chroniques sont tous nécessaires afin d'assurer que les Canadiens puissent vivre une vie longue, saine et exempte de maladies.

\section{Conflits d'intérêts}

Les auteures n’ont aucun conflit d'intérêts à déclarer.

\section{Contributions des auteures et avis}

LP a présidé le Comité directeur et a rédigé l'ébauche de l'aperçu, BB a géré le projet et analysé les données, PDR, PL, SO et GW ont analysé et interprété les données et toutes les coauteures ont revu et révisé l'aperçu.

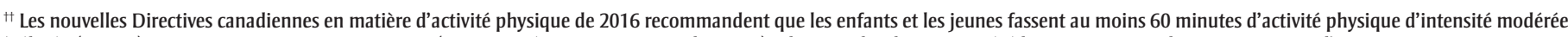

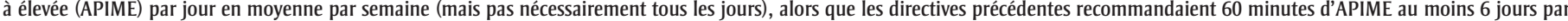

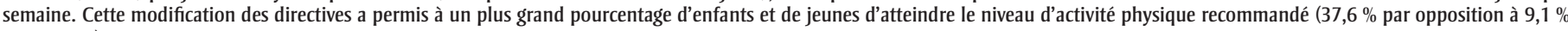
auparavant).
} 
Le contenu de l'article et les points de vue qui y sont exprimés n'engagent que les auteures; ils ne correspondent pas nécessairement à ceux du gouvernement du Canada.

\section{Références}

1. Agence de la santé publique du Canada. Quel est l'état de santé des Canadiens? Analyse des tendances relatives à la santé des Canadiens du point de vue des modes de vie sains et des maladies chroniques [Internet]. Ottawa (Ont.) : Agence de la santé publique du Canada; 2016. En ligne à : https://www.canada.ca/fr/sante -publique/services/publications/vie -saine/quel-est-l-etat-sante-des -canadiens.html

2. Organisation mondiale de la Santé. Global action plan for the prevention and control of noncommunicable diseases 2013-2020 [Internet]. Genève (Suisse) : Organisation mondiale de la Santé; 2013. En ligne à : http://www .who.int/nmh/events/ncd_action_ plan/en/ [en anglais seulement].

3. Société canadienne de physiologie de l'exercice. Directives canadiennes en matière de mouvements sur 24 heures pour les enfants et les jeunes : une approche intégrée regroupant l'activité physique, le comportement sédentaire et le sommeil [Internet]. Ottawa (Ont.) : Société canadienne de physiologie de l'exerice; 2016. En ligne à : https:// indd.adobe.com/view/7197c3fc-0a7b -4298-81ec-d048b49752b6 


\section{TABLEAU 1}

INDICATEURS DES MALADIES CHRONIQUES AU CANADA

STATISTIQUES RAPIDES, ÉDITION 2018

\begin{tabular}{|c|c|c|c|}
\hline $\begin{array}{c}\text { GROUPE } \\
\text { D'INDICATEURS }\end{array}$ & MESURE(S) D'INDICATEUR & $\begin{array}{l}\text { DONNÉES LES } \\
\text { PLUS RÉCENTES }\end{array}$ & $\begin{array}{l}\text { SOURCE DE } \\
\text { DONNÉES } \\
\text { (ANNÉE) }\end{array}$ \\
\hline \multicolumn{4}{|c|}{ DÉTERMINANTS SOCIAUX ET ENVIRONNEMENTAUX } \\
\hline Éducation & \% de la population déclarant ne pas avoir terminé ses études secondaires, population de 20 ans et plus & $12,2 \%$ & ESCC (2016) \\
\hline Revenu & \% de la population vivant sous les seuils de faible revenu après impôt, population totale & $8,1 \%$ & ECR (2016) \\
\hline $\begin{array}{l}\text { Pauvreté chez } \\
\text { les enfants }\end{array}$ & \% d'enfants vivant sous les seuils de faible revenu après impôt, population de moins de 18 ans ${ }^{b}$ & $7,3 \%$ & ECR (2016) \\
\hline Emploi & $\begin{array}{l}\text { Taux de chômage annuel moyen (\% de la population ne travaillant pas pendant la période de référence), } \\
\text { population de } 15 \text { ans et plus }\end{array}$ & $6,3 \%$ & EPA (2017) \\
\hline $\begin{array}{l}\text { Sentiment } \\
\text { d'appartenance } \\
\text { à la communauté }\end{array}$ & $\begin{array}{l}\text { \% de la population déclarant éprouver un sentiment d'appartenance « très fort » ou « plutôt fort » à sa } \\
\text { communauté locale, population de } 12 \text { ans et plus }\end{array}$ & $68,8 \%$ & ESCC (2016) \\
\hline \multicolumn{4}{|c|}{ FACTEURS DE RISQUE ET DE PROTECTION POUR LA SANTÉ DES MÈRES ET DES ENFANTS } \\
\hline $\begin{array}{l}\text { Diabète pendant } \\
\text { la grossesse }\end{array}$ & Taux de femmes enceintes ayant un diabète diagnnostiqué (gestationnel ou préexistant) & $\begin{array}{r}90,5 \text { par } 1000 \\
\text { naissances totales }\end{array}$ & BDCP (2016) \\
\hline $\begin{array}{l}\text { Hypertension } \\
\text { pendant la } \\
\text { grossesse }\end{array}$ & Taux de femmes enceintes ayant une hypertension diagnostiquée (gestationnelle ou préexistante) & $\begin{array}{r}68,2 \text { par } 1000 \\
\text { naissances totales }{ }^{c}\end{array}$ & BDCP (2016) \\
\hline $\begin{array}{l}\text { Poids de la mère } \\
\text { pendant la } \\
\text { grossesse }\end{array}$ & $\begin{array}{l}\text { \% de femmes déclarant un gain de poids pendant la grossesse supérieur aux recommandations } \\
\text { de Santé Canada }\end{array}$ & $42,8 \%{ }^{\mathrm{d}}$ & ESCC (2016) \\
\hline $\begin{array}{l}\text { Naissance } \\
\text { prématurée }\end{array}$ & \% de nouveau-nés vivants dont l'âge gestationnel n’atteint pas 37 semaines révolues & $\begin{array}{r}8,2 \text { par } 100 \\
\text { naissances } \\
\text { vivantes }\end{array}$ & BDCP (2016) \\
\hline $\begin{array}{l}\text { Allaitement } \\
\text { maternel }\end{array}$ & $\begin{array}{l}\text { \% de femmes déclarant avoir nourri leur enfant exclusivement au sein pendant les six premiers mois ou plus, } \\
\text { femmes de } 15 \text { ans et plus }\end{array}$ & $30,9 \%$ & ESCC (2016) \\
\hline $\begin{array}{l}\text { Exposition à la } \\
\text { fumée secondaire }\end{array}$ & $\begin{array}{l}\text { \% des ménages avec enfants de moins de } 15 \text { ans déclarant que les enfants sont régulièrement exposés } \\
\text { à la fumée secondaire à la maison }\end{array}$ & $2,8 \%$ & ECTAD (2015) \\
\hline Violence familiale & $\begin{array}{l}\text { \% de la population déclarant avoir subi au moins une fois avant l'âge de } 15 \text { ans tout type d'agression } \\
\text { physique ou sexuelle et/ou d'exposition à la violence par un adulte (18 ans et plus)e, population } \\
\text { de } 15 \text { ans et plus }\end{array}$ & $34,1 \%{ }^{\mathrm{e}}$ & ESG (2014) \\
\hline \multicolumn{4}{|c|}{ FACTEURS DE RISQUE ET DE PROTECTION COMPORTEMENTAUX } \\
\hline $\begin{array}{l}\text { Mouvement } \\
\text { sur } 24 \text { heures }\end{array}$ & $\begin{array}{l}\text { \% d'enfants et de jeunes qui suivent les Directives canadiennes en matière de mouvement } \\
\text { sur } 24 \text { heures à l'intention des enfants et des jeunes, population de } 5 \text { à } 17 \text { ans }\end{array}$ & $9,5 \%$ & $\begin{array}{r}\text { ECMS } \\
(2014 \text { à 2015) }\end{array}$ \\
\hline \multirow{2}{*}{ Activité physique } & $\begin{array}{l}\text { \% d'enfants et de jeunes qui suivent les recommandations en matière d'activité physique en pratiquant au } \\
\text { moins } 60 \text { minutes d'activité physique d'intensité modérée à élevée chaque jour (donnée mesurée), } \\
\text { population de } 5 \text { à } 17 \text { ans }\end{array}$ & $37,6 \%{ }^{\mathrm{d}}$ & $\begin{array}{r}\text { ECMS } \\
(2014 \text { à 2015) }\end{array}$ \\
\hline & $\begin{array}{l}\text { \% d'adultes qui suivent les recommandations en matière d'activité physique en pratiquant au moins } 150 \\
\text { minutes d'activité physique d'intensité modérée à élevée chaque semaine, en séances de } 10 \text { minutes ou plus } \\
\text { (donnée mesurée), population de } 18 \text { ans et plus }\end{array}$ & $17,5 \%$ & $\begin{array}{r}\text { ECMS } \\
(2014 \text { à 2015) }\end{array}$ \\
\hline $\begin{array}{l}\text { Comportement } \\
\text { sédentaire }\end{array}$ & $\begin{array}{l}\text { \% d'enfants et de jeunes déclarant suivre les recommandations en matière de comportement sédentaire en } \\
\text { consacrant moins de deux heures de loisir par jour à regarder la télévision ou à utiliser un ordinateur, } \\
\text { population de } 5 \text { à } 17 \text { ans }\end{array}$ & $28,5 \%{ }^{d}$ & $\begin{array}{r}\text { ECMS } \\
(2014 \text { à 2015) }\end{array}$ \\
\hline Sommeil & $\begin{array}{l}\text { \% de la population déclarant dormir le nombre d'heures recommandées chaque jour, } \\
\text { population de } 5 \text { ans et plus }\end{array}$ & $65,5 \%$ & $\begin{array}{r}\text { ECMS } \\
(2014 \text { à 2015) }\end{array}$ \\
\hline \multirow{2}{*}{ Alimentation } & $\begin{array}{l}\% \text { de la population déclarant consommer des fruits et des légumes au moins } 5 \text { fois par jour, } \\
\text { population de } 12 \text { ans et plus }\end{array}$ & $30,0 \%$ & ESCC (2016) \\
\hline & \% d'enfants et de jeunes déclarant boire des boissons sucrées tous les jours, population de 5 à 17 ans & $16,0 \%$ & $\begin{array}{r}\text { ECMS } \\
(2014 \text { à 2015) }\end{array}$ \\
\hline Stress chronique & $\begin{array}{l}\text { \% de la population déclarant que la vie est « assez » ou « extrêmement » stressante la plupart des jours au } \\
\text { cours des } 12 \text { derniers mois, population de } 12 \text { ans et plus }\end{array}$ & $21,5 \%$ & ESCC (2016) \\
\hline $\begin{array}{l}\text { Consommation } \\
\text { d'alcool }\end{array}$ & $\begin{array}{l}\text { \% de la population déclarant boire davantage d'alcool que ce que préconisent les directives de consomma- } \\
\text { tion d'alcool à faible risque à long terme, population de } 15 \text { ans et plus }\end{array}$ & $15,2 \%$ & ECTAD (2015) \\
\hline Tabagisme & $\%$ de la population déclarant fumer (quotidiennement ou occasionnellement), population de 15 ans et plus & $13,0 \%$ & ECTAD (2015) \\
\hline $\begin{array}{l}\text { Consommation } \\
\text { de drogues }\end{array}$ & $\begin{array}{l}\text { \% de la population déclarant avoir consommé du cannabis au moins une fois par semaine au cours des trois } \\
\text { derniers mois, population de } 15 \text { ans et plus }\end{array}$ & $5,2 \%$ & ECTAD (2015) \\
\hline $\begin{array}{l}\text { Prévalence des } \\
\text { principaux } \\
\text { facteurs de risque } \\
\text { de maladie } \\
\text { chronique }\end{array}$ & $\begin{array}{l}\text { \% de la population déclarant présenter au moins un des quatre principaux facteurs de risque de maladie } \\
\text { chronique (tabagisme, sédentarité, mauvaise alimentation et consommation nocive d'alcool), } \\
\text { population de } 20 \text { ans et plus }\end{array}$ & $84,9 \%$ & ESCC (2016) \\
\hline
\end{tabular}

chronique 


\begin{tabular}{|c|c|c|c|}
\hline $\begin{array}{c}\text { GROUPE } \\
\text { D'INDICATEURS }\end{array}$ & MESURE(S) D'INDICATEUR & $\begin{array}{l}\text { DONNÉES LES } \\
\text { PLUS RÉCENTES }\end{array}$ & $\begin{array}{l}\text { SOURCE DE } \\
\text { DONNÉES } \\
\text { (ANNÉE) }\end{array}$ \\
\hline \multicolumn{4}{|c|}{ FACTEURS DE RISQUE INTERMÉDIAIRES } \\
\hline \multirow{2}{*}{ Obésité } & \% d'enfants et de jeunes qui sont obèses (donnée mesurée), population de 5 à 17 ans & $13,1 \%$ & $\begin{array}{r}\text { ECMS } \\
(2014 \text { à 2015) }\end{array}$ \\
\hline & \% d'adultes qui sont obèses (donnée mesurée), population de 18 ans et plus & $28,1 \%$ & $\begin{array}{r}\text { ECMS } \\
\text { (2014 à 2015) }\end{array}$ \\
\hline Glycémie élevée & \% de la population ayant une glycémie élevée ${ }^{f}$ (donnée mesurée), population de 18 ans et plus & $4,1 \%$ & $\begin{array}{r}\text { ECMS } \\
\text { (2014 à 2015) }\end{array}$ \\
\hline $\begin{array}{l}\text { Cholestérol } \\
\text { sanguin élevé }\end{array}$ & $\begin{array}{l}\text { \% de la population ayant un taux élevéf de cholestérol (ratio cholestérol total sur cholestérol } \\
\text { LHD [CT:C-LHD]) (donnée mesurée), population de } 18 \text { ans et plus }\end{array}$ & $18,7 \%$ & $\begin{array}{r}\text { ECMS } \\
\text { (2014 à 2015) }\end{array}$ \\
\hline Hypertension & $\%$ de la population avec hypertension diagnostiquée, population de 20 ans et plus & $25,4 \%$ & SCSMC $(2015-16)^{8}$ \\
\hline \multicolumn{4}{|c|}{ PRATIQUES PRÉVENTIVES } \\
\hline \multirow{2}{*}{$\begin{array}{l}\text { Contact avec un } \\
\text { professionnel de } \\
\text { la santé }\end{array}$} & $\%$ de la population déclarant avoir un fournisseur habituel de soins de santé, population de 12 ans et plus & $84,2 \%$ & ESCC (2016) \\
\hline & $\begin{array}{l}\text { \% de la population déclarant avoir consulté un dentiste, un hygiéniste dentaire ou un orthodontiste au } \\
\text { moins une fois au cours des } 12 \text { derniers mois, population de } 12 \text { ans et plus }\end{array}$ & $69,3 \%$ & ESCC (2016) \\
\hline \multirow{3}{*}{$\begin{array}{l}\text { Dépistage des } \\
\text { maladies }\end{array}$} & $\begin{array}{l}\text { \% de femmes déclarant avoir eu au moins } 1 \text { mammographie au cours des } 5 \text { dernières années, population de } \\
50 \text { à } 74 \text { ans }\end{array}$ & $83,5 \%$ & ESCC (2012) \\
\hline & $\begin{array}{l}\% \text { de femmes déclarant avoir eu au moins } 1 \text { test PAP au cours des } 3 \text { dernières années, population de } 25 \text { à } 69 \\
\text { ans }\end{array}$ & $79,7 \%$ & ESCC (2012) \\
\hline & $\begin{array}{l}\text { \% de la population déclarant avoir eu au moins } 1 \text { test de recherche de sang occulte dans les selles, une } \\
\text { colonoscopie ou une sigmoïdoscopie au cours de la période recommandée, population de } 50 \text { à } 74 \text { ans }\end{array}$ & $51,1 \%$ & ESCC (2012) \\
\hline $\begin{array}{l}\text { Vaccination } \\
\text { (grippe) }\end{array}$ & $\begin{array}{l}\% \text { de la population atteinte d'un problème d'une maladie chronique }{ }^{\mathrm{h}} \text { déclarant avoir reçu un vaccin } \\
\text { antigrippal saisonnier au cours des } 12 \text { derniers mois, population de } 12 \text { ans et plus }\end{array}$ & $47,0 \%$ & ESCC (2016) \\
\hline \multicolumn{4}{|c|}{ ÉTAT DE SANTÉ GLOBAL ET IMPACTS SUR LA SANTÉ } \\
\hline \multirow{6}{*}{ Santé générale } & \% de la population déclarant avoir une « très bonne » ou une « excellente » santé, population de 12 ans et plus & $60,9 \%$ & ESCC (2016) \\
\hline & $\begin{array}{l}\text { \% de la population déclarant avoir une « très bonne » ou une « excellente » santé mentale, } \\
\text { population de } 12 \text { ans et plus }\end{array}$ & $68,5 \%$ & ESCC (2016) \\
\hline & Espérance de vie à la naissance & 83,3 ans & $\begin{array}{r}\text { SCSMC (2013-14 à } \\
2015-16)\end{array}$ \\
\hline & Espérance de vie à 65 ans & 21,8 ans & $\begin{array}{r}\text { SCSMC (2013-14 à } \\
2015-16)\end{array}$ \\
\hline & Espérance de vie ajustée en fonction de l'état de santé à la naissance & 71,9 ans & $\begin{array}{r}\text { SCSMC (2013-14 à } \\
2015-16)\end{array}$ \\
\hline & Espérance de vie ajustée en fonction de l'état de santé à 65 ans & 16,6 ans & $\begin{array}{r}\text { SCSMC (2013-14 à } \\
2015-16)\end{array}$ \\
\hline \multirow{15}{*}{$\begin{array}{l}\text { Morbidité } \\
\text { - prévalence }\end{array}$} & $\%$ de la population ayant un diabète diagnostiqué, population de 1 an et plus & $8,6 \%$ & SCSMC (2015-16) \\
\hline & $\begin{array}{l}\text { \% de la population déclarant avoir un diagnostic de maladies cardiovasculaires (maladie cardiaque ou } \\
\text { accident vasculaire cérébral), population de } 20 \text { ans et plus }\end{array}$ & $6,2 \%$ & ESCC (2016) \\
\hline & $\%$ de la population ayant une cardiopathie ischémique diagnostiquée, population de 20 ans et plus & $8,5 \%$ & SCSMC (2015-16) \\
\hline & $\%$ de la population ayant une insuffisance cardiaque diagnostiquée, population de 40 ans et plus & $3,7 \%$ & SCSMC (2015-16) \\
\hline & \% de la population ayant subi un accident vasculaire cérébral diagnostiqué, population de 20 ans et plus & $2,8 \%$ & SCSMC (2015-16) \\
\hline & $\%$ de la population ayant de l'asthme diagnostiqué, population de 1 an et plus & $11,4 \%$ & SCSMC (2015-16) \\
\hline & $\begin{array}{l}\% \text { de la population ayant une maladie pulmonaire obstructive chronique diagnostiquée, } \\
\text { population de } 35 \text { ans et plus }\end{array}$ & $10,2 \%$ & SCSMC (2015-16) \\
\hline & \% de la population déclarant avoir déjà reçu un diagnostic de cancer, population de 12 ans et plus & $7,2 \%$ & ESCC (2016) \\
\hline & $\begin{array}{l}\text { \% de la population déclarant avoir déjà eu des symptômes compatibles avec au moins une des six maladies } \\
\text { mentales ou troubles liés à l'utilisation de substances psychoactives', population de } 15 \text { ans et plus }\end{array}$ & $33,3 \%$ & ESCC-SM (2012) \\
\hline & $\begin{array}{l}\text { \% de la population déclarant avoir reçu un diagnostic de trouble de l'humeur ou de trouble anxieux, } \\
\text { population de } 12 \text { ans et plus }\end{array}$ & $12,9 \%$ & ESCC (2016) \\
\hline & $\begin{array}{l}\% \text { de la population ayant une démence diagnostiquée, y compris la maladie d'Alzheimer, } \\
\text { population de } 65 \text { ans et plus }\end{array}$ & $6,9 \%$ & SCSMC (2015-16) \\
\hline & $\%$ de la population ayant de l'ostéoarthrite diagnostiquée, population de 20 ans et plus & $13,7 \%$ & SCSMC (2015-16) \\
\hline & $\%$ de la population ayant de l'ostéoporose diagnostiquée, population de 40 ans et plus & $11,9 \%$ & SCSMC (2015-16) \\
\hline & $\begin{array}{l}\text { \% de la population déclarant avoir reçu un diagnostic d'au moins une des cinq maladies chroniques } \\
\text { communes', population de } 20 \text { ans et plus }\end{array}$ & $33,7 \%$ & ESCC (2016) \\
\hline & $\begin{array}{l}\text { \% de la population déclarant avoir reçu un diagnostic d'au moins une des } 10 \text { maladies chroniques } \\
\text { communesk } \text {, population de } 20 \text { ans et plus (Nouveau) }\end{array}$ & $44,2 \%$ & ESCC (2016) \\
\hline \multirow{2}{*}{ Multimorbidité } & $\begin{array}{l}\text { \% de la population déclarant avoir reçu un diagnostic d'au moins deux des cinq maladies chroniques } \\
\text { communes', population de } 20 \text { ans et plus }\end{array}$ & $8,9 \%$ & ESCC (2016) \\
\hline & $\begin{array}{l}\text { \% de la population déclarant avoir reçu un diagnnostic d'au moins deux des } 10 \text { maladies chroniques } \\
\text { communesk, population de } 20 \text { ans et plus }\end{array}$ & $18,3 \%$ & ESCC (2016) \\
\hline
\end{tabular}




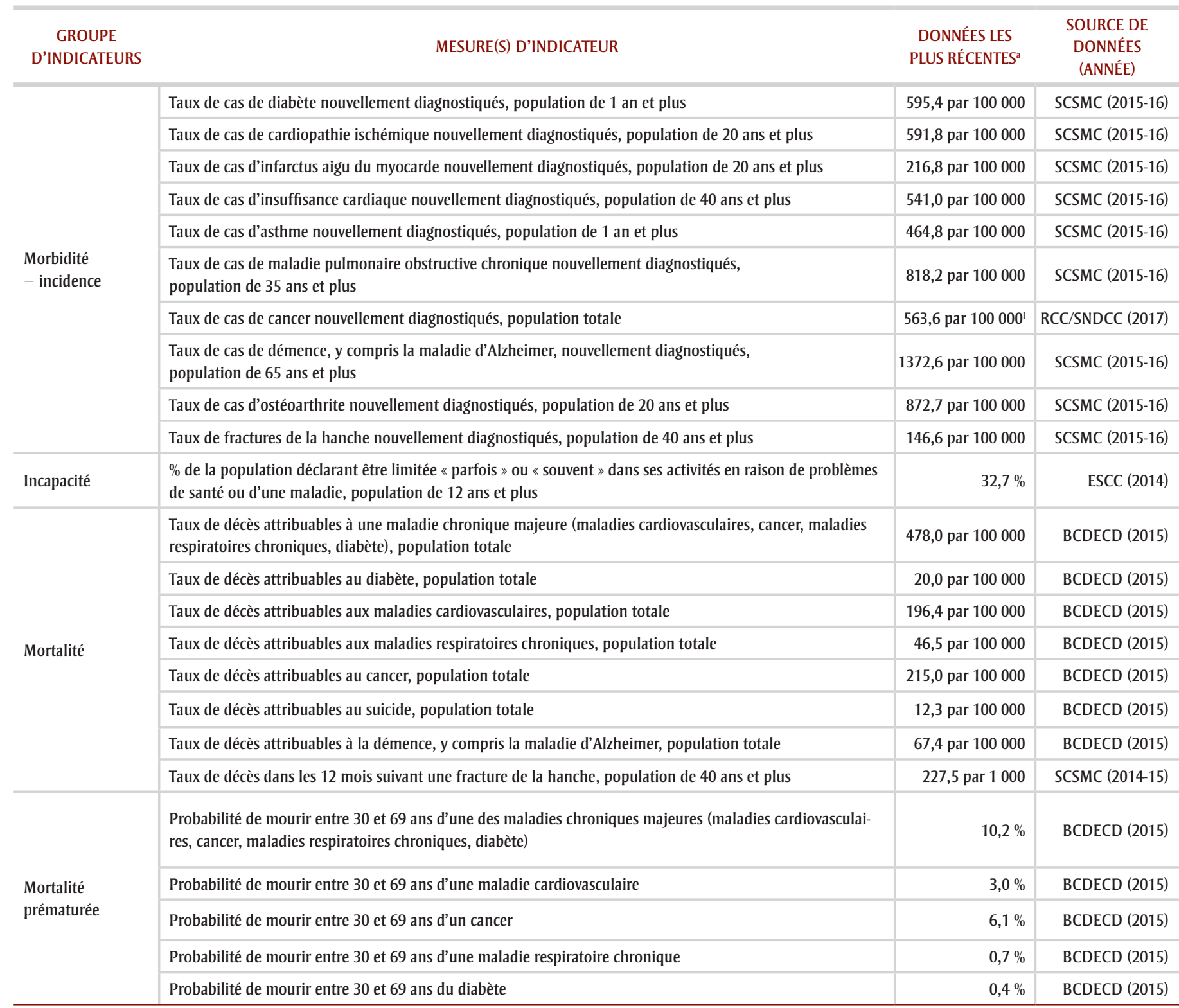

Abréviations : BCDECD, Statistiques de l'état civil - Base de données sur les décès; BDCP, Base de données sur les congés des patients; C-LHD, cholestérol à lipoprotéines de haute densité; CT, cholestérol total; ECMS, Enquête canadienne sur les mesures de la santé; ECR, Enquête canadienne sur le revenu; ECTAD, Enquête canadienne sur le tabac, l'alcool et les drogues; EPA, Enquête su cholestérol total; ECMS, Enquête canadienne sur les mesures de la santé; ECR, Enquête canadienne sur le revenu; ECTAD, Enquête canadienne sur le tabac, l'alcool et les drogues; EPA, Enquête sur la population active; ESCC, Enquête sur la santé dans les collectivités canadiennes; ESCC-SM, Enquête sur la santé dans les collectivités canadiennes-santé mentale; ESG, Enquête sociale générale; Remarque : Indicateurs ou mesures pour lesquels les données ne sont pas disponibles : tabagisme prénatal, consommation prénatale d’alcool, troubles du développement (y compris le trouble du spectre de l'autisme [TSA] et l'ensemble des troubles causés par l'alcoolisation foetale [ETCAF]), soutien social, résilience, discrimination et stigmatisation et environnement bâti.

${ }^{a}$ Tous les taux figurant dans ce tableau sont bruts et sont fondés sur des données réelles, à moins d'indication contraire.

${ }^{b}$ Inclut tous les enfants de 0 à 17 ans vivant dans une famille économique et ne vivant pas dans une famille économique.

'Les naissances totales incluent les naissances vivantes et les mortinaissances.

¿ La méthode de calcul utilisée pour cet indicateur diffère de celle des éditions précédentes, les estimations ne sont pas directement comparables.

e L'agression physique comprend avoir été giflé/frappé/poussé/attrapé/bousculé/projeté/attaqué physiquement au moins une fois par un adulte (18 ans et plus); l'agression sexuelle comprend avoir été forcé ou presque forcé à avoir une activité sexuelle/touché contre sa volonté d'une manière sexuelle au moins une fois par un adulte (18 ans et plus); et l'exposition à la violence comprend avoir vu ou entendu ses parents ou tuteurs se frapper ou frapper un autre adulte (18 ans et plus). La définition de cet indicateur a changé; les estimations ne sont pas directement comparables aux estimations précédemment rapportées.

${ }^{\mathrm{f}}$ Cet indicateur identifie les individus (à l'exception des femmes enceintes) avec un niveau élevé de cette condition à risque mesuré lors d'un prélèvement unique, sans toutefois tenir compte du statut diagnostique.

$\$$ Des données de l'ECMS existent pour cet indicateur. Elles présentent les taux pancanadiens d'hypertension artérielle selon le diagnostic.

h Les maladies chroniques incluent: le cancer (ayant reçu un diagnnostic dans le passé), le diabète, les maladies cardiovasculaires (maladies cardiaques et accidents vasculaires cérébraux), les maladies respiratoires chroniques (asthme et maladie pulmonaire obstructive chronique).

'Les six troubles mentaux et troubles liés à l'utilisation de substances sont : épisode dépressif majeur, trouble bipolaire, trouble d'anxiété généralisée et abus/dépendance à l'alcool, au cannabis ou à d'autres substances.

'Les cinq principaux groupes de maladies chroniques sont le cancer (ayant reçu un diağnostic dans le passé), le diabète, les maladies cardiovasculaires (maladies cardiaques et accidents vasculaires cérébraux), les maladies respiratoires chroniques (asthme et maladie pulmonaire obstructive chronique) et les troubles anxieux et/ou de l'humeur.

k Les dix maladies chroniques sont les maladies du cœeur, les accidents vasculaires cérébraux, le cancer (ayant reçu un diagnostic dans le passé), l'asthme, la maladie pulmonaire obstructive chronique, le diabète, l'arthrite, la maladie d'Alzheimer et autres démences, les troubles de l'humeur et/ou les troubles anxieux.

' Ces chiffres sont des projections pour 2017 fondées sur le fichier maître des totalisations du RCC d'août 2015 (données de 1992 à 2013) et le SNDCC (données de 1969 à 1991).

Citation suggérée : Agence de la santé publique du Canada. Indicateurs des maladies chroniques au Canada, Statistiques rapides, édition 2018. Ottawa (Ont.) : Agence de la santé publique du Canada; 2018. \#IMCC

Pour toutes questions ou commentaires, contactez-nous à : Infobase@phac-aspc.gc.ca

Utilisez l'outil en ligne des Indicateurs des maladies chroniques au Canada afin de voir des ventilations additionnelles de données (p. ex. selon le sexe, tendances au fil du temps) : http://infobase.phac-aspc.gc.ca. 